\title{
HANDBOOK OF FRUITS AND FRUIT PROCESSING
}




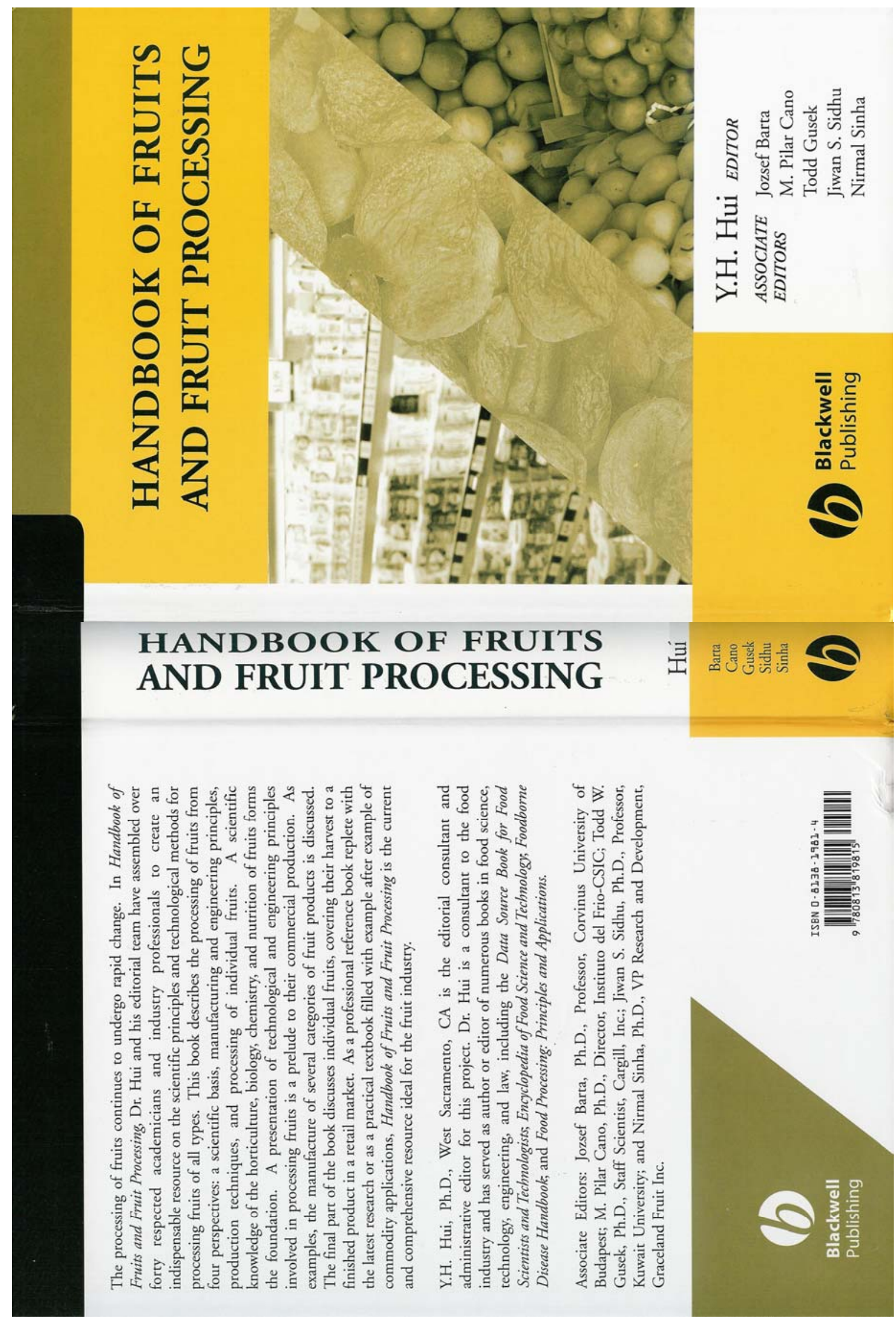




\title{
Handbook of Fruits and Fruit Processing
}

\author{
Editor \\ Y. H. Hui \\ Associate Editors \\ József Barta, M. Pilar Cano, Todd W. Gusek, \\ Jiwan S. Sidhu, and Nirmal Sinha
}


(C) 2006 Blackwell Publishing

All rights reserved

Blackwell Publishing Professional

2121 State Avenue, Ames, Iowa 50014, USA

Orders: $\quad 1-800-862-6657$

Office: $\quad 1-515-292-0140$

Fax: $\quad 1-515-292-3348$

Web site: www.blackwellprofessional.com

Blackwell Publishing Ltd

9600 Garsington Road, Oxford OX4 2DQ, UK

Tel.: +44 (0)1865 776868

Blackwell Publishing Asia

550 Swanston Street, Carlton, Victoria 3053, Australia Tel.: +61 (0)383591011

Authorization to photocopy items for internal or personal use, or the internal or personal use of specific clients, is granted by Blackwell Publishing, provided that the base fee of $\$ .10$ per copy is paid directly to the Copyright Clearance Center, 222 Rosewood Drive,
Danvers, MA 01923. For those organizations that have been granted a photocopy license by CCC, a separate system of payments has been arranged. The fee codes for users of the Transactional Reporting Service are ISBN-13: 978-0-8138-1981-5; ISBN-10: 0-8138$1981-4 / 2006 \$ .10$.

First edition, 2006

Library of Congress Cataloging-in-Publication Data

Handbook of fruits and fruit processing / editor, Y.H. Hui; associate editors, József Barta ...

[et al.]. - 1st ed. p. $\mathrm{cm}$.

Includes index.

ISBN-13: 978-0-8138-1981-5 (alk. paper)

ISBN-10: 0-8138-1981-4 (alk. paper)

1. Food industry and trade. 2. Fruit-Processing. I. Hui, Y. H. (Yiu H.) II. Barta, József.

TP370.H264 2006

$664^{\prime} .8-\mathrm{dc} 22$ 


\section{Contents}

Contributors, vii

Preface, xi

\section{Part I Processing Technology}

1. Fruit Microbiology, 3

A. Kalia and R. P. Gupta

2. Nutritional Values of Fruits, 29

C. Sánchez-Moreno, S. De Pascual-Teresa, B. De Ancos, and M. P. Cano

3. Fruit Processing: Principles of Heat Treatment, 45

\section{Körmendy}

4. Fruit Freezing Principles, 59

B. De Ancos, C. Sánchez-Moreno, S. De Pascual-Teresa, and M. P. Cano

5. Fruit Drying Principles, 81

J. Barta

6. Non-Thermal Pasteurization of Fruit Juice Using High Voltage Pulsed Electric Fields, 95

Zs. Cserhalmi

7. Minimally Processed Fruits and Fruit Products and Their Microbiological Safety, 115

Cs. Balla and J. Farkas

8. Fresh-Cut Fruits, 129

O. Martín-Belloso, R. Soliva-Fortuny, and G. Oms-Oliu

9. Food Additives in Fruit Processing, 145

P. S. Raju and A. S. Bawa

10. Fruit Processing Waste Management, 171

J. Monspart-Sényi

\section{Part II Products Manufacturing}

11. Manufacturing Jams and Jellies, 189

H. S. Vibhakara and A. S. Bawa

12. Manufacturing Fruit Beverages, 205

E. Horváth-Kerkai

13. Fruit as an Ingredient in a Fruit Product, 217

Gy. Pátkai

14. Fruit Processing Plant, 231

J. Barta

15. Fruits: Sanitation and Safety, 245

S. Al-Zenki and H. Al-Omariah 


\section{Part III Commodity Processing}

16. Apples, 265

N. Sinha

17. Apricots, 279

M. Siddiq

18. Horticultural and Quality Aspects of Citrus Fruits, 293

M. J. Rodrigo and L. Zacarías

19. Oranges and Citrus Juices, 309

K. S. Sandhu and K. S. Minhas

20. Sweet Cherries, 359

J. Alonso and R. Alique

21. Cranberry, Blueberry, Currant, and Gooseberry, 369

K. K. Girard and N. Sinha

22. Date Fruits Production and Processing, 391

J. S. Sidhu

23. Grape Juice, 421

$O$. Martín-Belloso and A. R. Marsellés-Fontanet

24. Grapes and Raisins, 439

N. R. Bhat, B. B. Desai, and M. K. Suleiman

25. Grape and Wine Biotechnology: Setting New Goals for the Design of Improved Grapevines, Wine Yeast, and Malolactic Bacteria, 453

I. S. Pretorius

26. Olive Processing, 491

B. Gandul-Rojas and M. I. Mínguez-Mosquera

27. Peach and Nectarine, 519

M. Siddiq

28. Pear Drying, 533

R. de Pinho Ferreira Guiné

29. Plums and Prunes, 553

M. Siddiq

30. Processing of Red Pepper Fruits (Capsicum annuum L.) for Production of Paprika and Paprika Oleoresin, 565

A. Pérez-Gálvez, M. Jarén-Galán, and M. I. Mínguez-Mosquera

31. Strawberries and Raspberries, 581

N. Sinha

32. Tropical Fruits: Guava, Lychee, Papaya, 597

J. S. Sidhu

33. Banana, Mango, and Passion Fruit, 635

L. G. Occeña-Po

34. Nutritional and Medicinal Uses of Prickly Pear Cladodes and Fruits:

Processing Technology Experiences and Constraints, 651

M. Hamdi

35. Speciality Fruits Unique to Hungary, 665

M. Stéger-Máté

Index, 679 


\section{Contributors}

Rafael Alique (Chapter 20)

Instituto del Frío (CSIC)

C/José Antonio Novais $n^{\circ} 10$

28040 Madrid, Spain

Phone: +34915492300

Jesús Alonso (Chapter 20)

Instituto del Frío (CSIC)

C/José Antonio Novais n ${ }^{\circ} 10$

28040 Madrid, Spain

Phone: +34915492300

E-mail: jalonso@if.csic.es

Husam Al-Omariah (Chapter 15)

Biotechnology Department

Kuwait Institute for Scientific Research

P.O. Box 24885, 13109-Safat, Kuwait

Sameer Al-Zenki (Chapter 15)

Biotechnology Department

Kuwait Institute for Scientific Research

P.O. Box 24885, 13109-Safat, Kuwait

Phone: (965)-483-6100

Fax: (965)-483-4670

E-mail: szenki@kisr.edu.kw

Begoña De Ancos (Chapters 2, 4)

Department of Plant Foods Science

and Technology, Instituto del Frío

Consejo Superior de Investigaciones

Científicas (CSIC) Ciudad Universitaria

E-28040 Madrid, Spain

E-mail: ancos@if.csic.es
Csaba Balla (Chapter 7)

Corvinus University of Budapest, Faculty of Food Science, Department of Refrigeration and Livestock Products Technology

Hungary 1118, Budapest, Ménesi út 45

Phone: 36-1-482-6064

Fax: 36-1-482-6321

E-mail: csaba.balla@uni-corvinus.hu

József Barta, Ph.D. (Chapters 5, 14)

Head of the Department

Corvinus University of Budapest

Faculty of Food Science

Department of Food Preservation

Budapest, Ménesi út 45

Hungary 1118

Phone: 36-1-482-6212

Fax: 36-1-482-6327

E-mail: jozsef.barta@uni-corvinus.hu

A.S. Bawa (Chapters 9, 11)

Fruits and Vegetables Technology

Defence Food Research Laboratory

Siddarthanagar, Mysore-570 011, India

Phone: 0821-247-3783

Fax: 0821-247-3468

E-mail: dfoodlab@sancharnet.in

N. R. Bhat (Chapter 24)

Arid Land Agriculture Department

Kuwait Institute for Scientific Research

P.O. Box 24885, 13109-Safat, Kuwait

E-mail: nbhat@safat.kisr.edu.kw 
M. Pilar Cano, Ph.D. (Chapters 2, 4)

Director

Instituto del Frío-CSIC

C/Jose Antonio Novais, 10

Ciudad Universitaria

28040-Madrid, Spain

Phone: 34-91-5492300

Fax: 34-91-5493627

E-mail: pcano@if.csic.es

Zsuzsanna Cserhalmi (Chapter 6)

Central Food Research Institute

Hungary 1022 Budapest, Hermann O. u. 15

Phone: 36-1-214-1248

Fax: 36-1-355-8928

E-mail: zs.cserhalmi@cfri.hu

Sonia De Pascual-Teresa (Chapters 2, 4)

Department of Plant Foods Science

and Technology, Instituto del Frío

Consejo Superior de Investigaciones

Científicas (CSIC) Ciudad Universitaria

E-28040 Madrid, Spain

E-mail: soniapt@if.csic.es

B. B. Desai (Chapter 24)

Arid Land Agriculture Department

Kuwait Institute for Scientific Research

P.O. Box 24885, 13109-Safat, Kuwait

József Farkas (Chapter 7)

Corvinus University of Budapest

Faculty of Food Science, Department

of Refrigeration and Livestock Products

Technology and Central Food Research Institute

Hungary 1118, Budapest, Ménesi út 45

and, 1022, Budapest, Hermann O. u. 15

Phone: 36-1-482-6303

Fax: 36-1-482-6321

E-mail: j.farkas@cfri.hu

Raquel de Pinho Ferreira Guiné (Chapter 28)

Associate Professor

Department of Food Engineering

ESAV, Polytechnic Institute of Viseu

Campus Politécnico, Repeses

3504-510 Viseu, Portugal

E-mail: raquelguine@esav.ipv.pt
Beatriz Gandul-Rojas (Chapter 26)

Group of Chemistry and Biochemistry

of Pigments. Food Biotechnology Department

Instituto de la Grasa (CSIC).

Av. Padre García Tejero 4, 41012

Sevilla, Spain

Kristen K. Girard (Chapter 21)

Principal Scientist

Ocean Spray Cranberries, Inc.

Ingredients

1 Ocean Spray Dr.

Middleboro MA 02349, USA

E-mail: kgirard@oceanspray.com

Rajinder P. Gupta (Chapter 1)

Department of Microbiology,

College of Basic Sciences and Humanities

Punjab Agricultural University

Ludhiana-141004, India

rpguptag@rediffmail.com

Todd W. Gusek, Ph.D.

Principal Scientist, Central Research

Cargill, Inc.

PO Box 5699

Minneapolis, MN 55440, USA

Phone: (952)742-6523

Fax: (952)742-4925

E-mail: todd_gusek@cargill.com

M. Hamdi (Chapter 34)

Director, Department of Biochemical and Chemical

Engineering Microbial and Food Processes

Higher School of Food Industries

National Institute of Applied Sciences

and Technology. BP: 676. 1080 Tunisia

Phone: 216-98-326675

Fax: 216-71-704-329

E-mail: moktar.hamdi@insat.rnu.tn

Emoke Horváth-Kerkai (Chapter 12)

Corvinus University of Budapest, Faculty

of Food Science, Department of

Food Preservation Hungary 1118

Budapest, Ménesi út 45.

Phone: 36-1-482-6035

Fax: 36-1-482-6327

E-mail: emoke.kerkai@uni-corvinus.hu 
Y. H. Hui, Ph.D.

Senior Scientist

Science Technology System

P.O. Box 1374

West Sacramento, CA 95691, USA

Phone: 916-372-2655

Fax: 916-372-2690

E-mail: yhhui@aol.com

Manuel Jarén-Galán (Chapter 30)

Group of Chemistry and Biochemistry

of Pigments. Food Biotechnology Department

Instituto de la Grasa (CSIC)

Av. Padre García Tejero 4, 41012

Sevilla, Spain

Anu Kalia (Chapter 1)

Department of Microbiology,

College of Basic Sciences and Humanities

Punjab Agricultural University

Ludhiana-141004, India

kaliaanu@rediffmail.com

Imre Körmendy (Chapter 3)

Corvinus University of Budapest,

Faculty of Food Science, Department

of Food Preservation Hungary 1118

Budapest, Ménesi út 45

Phone: 36-1-482-6212

Fax: 36-1-482-6327

E-mail: imre.kormendy@uni-corvinus.hu

Olga Martín-Belloso (Chapters 8, 23)

Department of Food Technology, University

of Lleida Av. Alcalde Rovira Roure, 191. 25198

Lleida, Spain

Phone: +34-973-702-593

Fax: +34-973-702-596

E-mail: omartin@tecal.udl.es

Angel Robert Marsellés-Fontanet (Chapter 23)

Department of Food Technology, University

of Lleida Av. Alcalde Rovira Roure, 191. 25198

Lleida, Spain

Phone: +34 973702593

Fax: +34 973702596

E-mail: rmarselles@tecal.udl.es

M. Isabel Mínguez-Mosquera (Chapters 26, 30) Group of Chemistry and Biochemistry of Pigments. Food Biotechnology Department Instituto de la Grasa (CSIC)
Av. Padre García Tejero 4, 41012

Sevilla, Spain

Phone: +34954691054

Fax: +34954691262

E-mail:minguez@cica.es.

Kuldip Singh Minhas (Chapter 19)

Professor

Food Science and Technology

Punjab Agricultural University

Ludhiana, Punjab, India

Phone: 0161-2401960 Extn. 305

Judit Monspart-Sényi (Chapter 10)

Corvinus University of Budapest, Faculty

of Food Science, Department of Food Preservation

Hungary 1118, Budapest, Ménesi út 45

Phone: 36-1-482-6037

Fax: 36-1-482-6327

E-mail: judit.senyi@uni-corvinus.hu

Lillian G. Occeña-Po (Chapter 33)

Department of Food Science and Human Nutrition

Michigan State University

East Lansing, MI 48824, USA

Phone: 517-432-7022

Fax: 517-353-8963

E-mail: occena@msu.edu

Gemma Oms-Oliu (Chapter 8)

Department of Food Technology, University of

Lleida Av. Alcalde Rovira Roure, 191. 25198

Lleida, Spain

Phone: +34-973-702-593

Fax: +34-973-702-596

E-mail: goms@tecal.udl.es

Györgyi Pátkai (Chapter 13)

Corvinus University of Budapest, Faculty

of Food Science, Department of Food Preservation

Hungary 1118, Budapest, Ménesi út 45

Phone: 36-1-482-6212

Fax: 36-1-482-6327

E-mail: gyorgyi.patkai@uni-corvinus.hu

Antonio Pérez-Gálvez (Chapter 30)

Group of Chemistry and Biochemistry

of Pigments, Food Biotechnology Department

Instituto de la Grasa (CSIC).

Av. Padre García Tejero 4, 41012,

Sevilla, Spain 
Isak S. Pretorius (Chapter 25)

The Australian Wine Research Institute PO Box 197, Glen Osmond

Adelaide, SA 5064

Australia

Phone: $+61-8-83036835$

Fax: +61-8-83036601

E-mail: Sakkie.Pretorius@awri.com.au

P.S. Raju (Chapter 9)

Fruits and Vegetables Technology

Defence Food Research Laboratory

Siddarthanagar, Mysore-570 011, India

Phone: 0821-247-3783

Fax: 0821-247-3468

E-mail: dfoodlab@sancharnet.in

María Jesús Rodrigo (Chapter 18)

Instituto de Agroquímica y Tecnología de Alimentos (CSIC). Apartado Postal 73

46100 Burjasot, Valencia, Spain

Concepción Sánchez-Moreno (Chapters 2, 4)

Department of Plant Foods Science and

Technology, Instituto del Frío, Consejo Superior

de Investigaciones Científicas (CSIC)

Ciudad Universitaria, E-28040 Madrid, Spain

E-mail: csanchezm@if.csic.es

Kulwant S. Sandhu (Chapter 19)

Sr. Veg. Technologist (KSS)

Department of Food Science and Technology

Punjab Agricultural University

Ludhiana - 141 004, Punjab, India

Phone: 0161-2405257, 2401960 extn. 8478

(KSS)

E-mail: ptc@satyam.net.in

Jiwan S. Sidhu, Ph.D. (Chapters 22, 32)

Professor, Department of Family Science

College for Women, Kuwait University

P.O. Box 5969, Safat-13060, Kuwait

Phone: (965)-254-0100 extn. 3307

Fax: (965)-251-3929

E-mails: jsidhu@cfw.kuniv.edu;

jiwansidhu2001@yahoo.com

Muhammad Siddiq (Chapters 17, 27, 29)

Food Processing Specialist

Department of Food Science \& Human Nutrition

Michigan State University
East Lansing, MI 48824, USA

Phone: 517-355-8474

Fax: 517-353-8963

E-mail: siddiq@msu.edu

Nirmal Sinha, Ph.D. (Chapters 16, 21, 31)

VP, Research and Development

Graceland Fruit, Inc.

1123 Main Street

Frankfort, MI 49635, USA

Phone: 231-352-7181

Fax: 231-352-4711

E-mail: nsinha@gracelandfruit.com

Robert Soliva-Fortuny (Chapter 8)

Department of Food Technology, University

of Lleida Av. Alcalde Rovira Roure, 191. 25198

Lleida, Spain

Phone: +34-973-702-593

Fax: +34-973-702-596

E-mail: rsoliva@tecal.udl.es

Mónika Stéger-Máté (Chapter 35)

Corvinus University of Budapest, Faculty

of Food Science, Department of Food Preservation

Hungary 1118, Budapest, Ménesi út 45

Phone: 36-1-482-6034

Fax: 36-1-482-6327

E-mail: monika.stegernemate@uni-corvinus.hu

M. K. Suleiman (Chapter 24)

Arid Land Agriculture Department

Kuwait Institute for Scientific Research

P.O. Box 24885, 13109-Safat, Kuwait

H.S. Vibhakara (Chapter 11)

Fruits and Vegetables Technology

Defence Food Research Laboratory

Siddarthanagar, Mysore-570 011, India

Phone: 0821-247-3949

Fax: 0821-247-3468

Lorenzo Zacarías (Chapter 18)

Instituto de Agroquímica y Tecnología

de Alimentos (CSIC). Apartado Postal 73

46100 Burjasot, Valencia, Spain

Phone: 34963900022

Fax: 34963636301

E-mail: Izacarias@iata.csic.es or

cielor@iata.csic.es 